\title{
N-BODY SIMULATIONS OF THE MAGELLANIC SYSTEM INCLUDING GAS DYNAMICS AND STAR FORMATION
}

\author{
A.M. YOSHIZAWA AND M. NOGUCHI \\ Astronomical Institute, Tohoku University \\ Aoba, Sendai 980-77, Japan
}

The system of the Magellanic Clouds is considered to be dynamically interacting among themselves and with our Galaxy. This interaction is thought to be the cause of many complicated features seen in the Magellanic Clouds and the Magellanic Stream (see Westerlund 1990, $A \mathscr{E} A R, 2,27$ ). In order to better understand the formation and evolution of the Magellanic System, we carry out realistic N-body simulations of the tidal distortion of the Small Magellanic Cloud (SMC) due to our Galaxy and the Large Magellanic Cloud (LMC).

The present work is based on the tidal model (e.g., Murai \& Fujimoto 1980, PASJ, 32, 581; Gardiner \& Noguchi 1996, MNRAS, 278, 191). A novel point of this work is that it takes into account the motion of interstellar gas clouds and the star formation process. We succeeded in reproducing a gas stream with almost no stars as a result of the close encounter between the SMC and the LMC plus our Galaxy about 1.5 Gyr ago. We also reproduced the spatial distribution of gas particles around the Inter-Cloud Region and the star formation property in the center and the vicinity of the SMC. These features have been caused by the latest encounter with the LMC about 0.2 Gyr ago. The major achievement of this work is to provide a possibility of explaining observed structural, kinematic, and star formation properties of the SMC, and other related tidal features in the Magellanic System, without resorting to non-gravitational processes such as ram pressure due to the hot halo gas of our Galaxy or collisions of the SMC gas with the high velocity clouds. A detailed comparison of the model results with observational data would enable a deeper understanding of the gas dynamical processes and star formation activity in this nearest interacting galaxy system. A full paper will be published elsewhere. 